\title{
A Study of the Impact of Collaborative Problem-Solving Strategies on Students' Performance of Simulation-Based Learning - A Case of Network Basic Concepts Course
}

\author{
Hsin-Ke Lu and Peng-Chun Lin
}

\begin{abstract}
The continued development of the Internet has caused the concepts of network infrastructure and simple network troubleshooting to become important teaching objectives of certain college curricula. However, the abstract nature of network concepts and the high cost of equipment for network-related practice have become educational challenges for teachers in the relevant fields. Therefore, simulation learning and simulation learning software have become major instructional tools to assist teachers. Unfortunately, most simulation learning software is individually manipulated, which may cause feelings of isolation among students during the course of learning. Therefore, in this study, a quasi-experimental approach was utilized to investigate how teaching strategies for simulation learning and for integrating simulation learning with collaborative problem solving (CPS) impacted learning outcomes. Assessments by one-way analysis of covariance revealed that the post-test scores were significantly better in the experimental group than in the control group, suggesting that the integrated educational activities were beneficial to students' learning. The results of this study can serve as a reference for teachers in relevant fields with respect to designing educational activities that can help students learn more effectively and improve students' teamwork abilities.
\end{abstract}

Index Terms - Simulation-based learning, CPS, collaborative problem solving, information and computer education, packet tracer.

\section{INTRODUCTION}

Due to the rapid development of network technology, individuals' Internet use requirements are increasing daily. Colleges and universities generally offer courses that teach basic network-related concepts. These courses enhance students' abilities to use network technology by enabling students to understand the mode of operation of the Internet and allowing students to perform simple troubleshooting. However, Internet-based concepts, such as the principles underlying various communication protocols and packet-forwarding processes, have always been a relatively abstract knowledge system. Thus, teaching in this field has long been a challenge for instructors. In addition, the management of network devices is a skill that must be mastered through hands-on training. In particular, providing learners with opportunities to construct procedural knowledge and train in practical skills through hands-on activities are extremely important educational tools in science,

Manuscript received December 31, 2015; revised March 11, 2016.

The authors are with the Chinese Culture University, Taipei, Taiwan (e-mail: sklu@sce.pccu.edu.tw, sklu@sce.pccu.edu.tw). technology, engineering and mathematics (STEM) fields However, in actual educational environments, due to various considerations, including time, space and funding, it can be difficult for colleges and universities to provide students with adequate training in hands-on environments. Thus, the use of information technology to establish simulated operational environments plays an extremely important role in strengthening students' learning of procedural knowledge [1]

A main characteristic of computer-based simulation learning is the use of information technology to create models that simulate real-world environments. Many phenomena that are difficult to observe in real-world environments can be concretized by computer-based simulation learning. Moreover, the simulation system's judgments can be used to present material in a manner appropriate to a learner's knowledge level, thereby helping students better understand these phenomena. Thus, simulation learning can safely and inexpensively provide students with environments similar to real-life situations. As a result, students can learn about relevant issues through an educational process involving exploration and constant attempts at experimentation and can thereby acquire a deeper understanding of the simulated phenomenon through hands-on manipulation [2]. Therefore, for educators, relevant software for simulation learning has become an important tool for providing teaching assistance. Many scholars have previously proposed different instructional-design approaches for enhancing students' learning outcomes in the information technology and network fields, and research has proven that simulation-based learning can effectively improve learning outcomes [3], [4].

However, in a simulated learning environment, most support tools mainly allow for manipulation and learning only by an individual learner. One such tool is "Think Tool," which was designed by White (1993) and White and Frederiksen (1998) to assist students in learning the abstract concepts of physical mechanics or to provide teachers with an easy Java simulation (EJS) design environment for the design and production of computer-based scientific simulations and animations [5], [6]. However, the use of simulated learning environments that are manipulated by only an individual learner may generate relevant concerns regarding isolated learning. Vygotsky (1978) believed that learning and development are not independent but are instead mutually influential processes. From the perspective of cognitive elaboration theory, learners can incorporate knowledge through cognitive restructuring and mastery processes (such as summarizing and restating, among other processes) that allow newly learned information to be retained in the memory 
and connected with prior experiences [7]. A student who explains newly learned information to others must organize and interpret the information in question; thus, explanations to others may not only promote the refinement of the explainer's own perceptions but may also improve the outcomes of cooperative learning activities [8]. Therefore, cooperative learning is a common teaching strategy in STEM fields. Roth and Roychoudury (1993) stated that cooperative learning can improve students' academic performance, enhance comprehension and problem-solving abilities, and re-construct or alter the conceptual frameworks of students' knowledge through processes of communication and discussion [9].

Therefore, in this study, a quasi-experimental approach was utilized to examine groups of college students who were obtaining a basic introduction to networks to investigate whether the use of network-simulation software could enhance students' learning outcomes. Moreover, the question of whether an instructional design that integrated network educational-simulation software with collaborative problem-solving (CPS) strategies could further improve students' learning outcomes was also explored.

\section{LITERATURE REVIEW}

\section{A. Simulation-Based Learning}

As technology has advanced, the integration of computer-based instruction methods into education has become an increasingly widespread trend. In particular, learning through simulation and manipulation plays an extremely important role in education in STEM fields. Bransford, Brown and Cocking (2000) noted that good learning outcomes can be achieved if students construct their own understanding of scientific knowledge within existing knowledge frameworks. To accomplish this objective, students must proactively participate in and learn from the educational process [10]; interactive computer simulation software can help to meet this requirement [11]. During the course of teaching in scientific education, dynamic and interactive computer simulations can improve students' learning outcomes with respect to scientific phenomena that are difficult to observe in a real-world context [12]. By enabling repeated manipulations or observations, the use of simulation software can help learners acquire a deeper understanding of scientific phenomena and appropriately promote implementation, reflective competence and high-level cognition for the relevant procedural knowledge. Studies have also determined that virtual manipulations in software-based simulation environments can contribute to a greater understanding of domain knowledge [12], [13].

From the perspective of teaching, the main purpose of utilizing computer-based simulation and manipulation is to meet the needs of learners by providing opportunities to practice the solving of real-world problems [14]. Students often cannot attempt to address these problems in real-world environments due to limitations imposed by various factors, such as time, cost and risk; fortunately, these situations can be successfully modeled through computer simulation [15]. Many studies have utilized simulation-based software to assist with diverse aspects of scientific instruction, including physics experiments [16]-[18], molecular dynamics [19], and knowledge of circuits [20]; these studies have found that the use of simulation software has positive effects on all levels to enhance learning.

The network simulation-based learning tool(Packet Tracer) used in this study, which was developed by Cisco Systems, was specifically designed to illustrate and teach network concepts and skills of network equipment operations. A graphical user interface with real-time feedback allows students to design and simulate real-world network traffic, thereby enabling students to learn an abstract knowledge system that is typically difficult to observe. According to Frezzo et al. (2009), the use of Packet Tracer as an instructional tool can provide students with a relatively structured and logical learning environment [21]. Many studies have also mentioned that the integration of Packet Tracer into teaching can enhance students' interest in learning and improve learning outcomes [3], [21], [22].

\section{B. Collaborative Problem Solving (CPS)}

In STEM fields, a great deal of knowledge must be learned through manipulation and the process of attempting to solve problems by troubleshooting. Therefore, the learning of procedural knowledge is extremely important. Procedural knowledge refers to knowledge obtained during the process of performing operations and the ability to utilize information to solve problems. Frequently, certain types of activity are presumably necessary to indirectly derive procedural knowledge by effectively practicing how to approach problems and what methods to utilize. Procedural knowledge mainly includes the methods, techniques, strategies, procedures and steps of solving problems. Problem-solving strategies are often applied in courses in relevant fields for the teaching and learning of procedural knowledge, and researchers have explored students' learning processes and learning outcomes [23]-[26]. Problem solving is a widely adopted teaching strategy [23], and many studies have explored problem-solving based teaching strategies involving the use of technologies. Numerous studies have also stated that cooperative learning can effectively improve learning performance for students of all ages. Therefore, Nelson (1999) proposed CPS, which integrates the two approaches of cooperative learning and problem-based learning. CPS encourages students to learn by doing and stresses the authenticity of the collaborative learning environment; in CPS, students become participants in active learning processes, with an emphasis on independent thinking and problem-solving capabilities [27]. In the CPS pedagogy, objectives include not only the development of problem-solving skills but also guiding the development of learners' cooperation and communication abilities. In a heterogeneous group, the abilities and perspectives of an individual are limited; thus, to find the appropriate solution for a problem, there must be cooperation among group members and even those with differing views [28], [29]. Therefore, for the experimental group of this study, in addition to using Packet Tracer to assist students in learning basic networking concepts, CPS strategy was also integrated into the instructional approach to investigate whether this 
integration could enhance students' learning outcomes.

\section{RESEARCH METHOD}

This study used a quasi-experimental approach to investigate the learning outcomes of universities' students who were enrolled in a semester course that provided a basic competence to networks.

\section{A. Experimental Design}

In this study, given the characteristics of the experimental setting, one class of students was randomly designated as the experimental group, and another class was designated as the control group for comparison analysis. For both groups of students, the Packet Tracer simulator and assessment questionnaire were used as an instructional setting to facilitate learning. In the experimental group, the baseline learning activities were integrated with activities designed using CPS strategies; these CPS activities allowed students to communicate and learn through group discussions. A pre-test on the network competency was administered prior to the learning activities, and a post-test on the network competency was administered after the completion of these activities. The research framework of this study can be depicted as follows (Fig. 1):

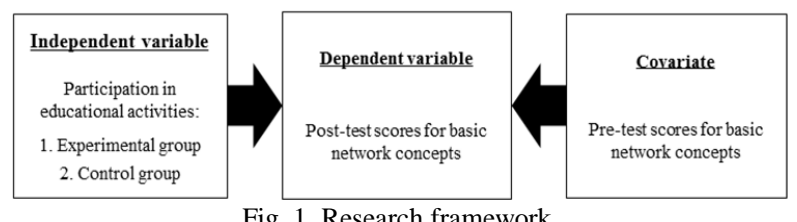

Fig. 1. Research framework.

The independent variable in this study was participation in learning activities, the dependent variable was the post-test scores of the experimental and control group students on the "Basic Network Concept Inventory (BNCI)," and the covariate was the pre-test scores of experimental and control group students. The experimental design is presented in Table I. G1 refers to experimental group students, whereas G2 refers to control group students; $\mathrm{O} 1$ and $\mathrm{O} 3$ refer to the pre-test scores of the experimental and control group students, respectively, on the BNCI. The independent variable was involvement in learning activities, where $\mathrm{X}(\mathrm{CPS})$ represents the experimental treatment of $\mathrm{CPS} ; \mathrm{O} 2$ and $\mathrm{O} 4$ refer to the study's dependent variable, the post-test scores of the experimental and control group students, respectively, on the BNCI after the implementation of learning activities.

TABLE I: PRE- AND POST-TEST QUASI-EXPERIMENTAL DESIGN

\begin{tabular}{lccc}
\hline \hline \multicolumn{1}{c}{ Group } & Pre-test & $\begin{array}{c}\text { Experimental } \\
\text { treatment }\end{array}$ & Post-test \\
\hline Experimental group (G1) & $\mathrm{O}_{1}$ & $\mathrm{X}(\mathrm{CPS})$ & $\mathrm{O}_{2}$ \\
Control group (G2) & $\mathrm{O}_{3}$ & & $\mathrm{O}_{4}$ \\
\hline \hline
\end{tabular}

The learning activities involved the teacher's design of network scenarios for troubleshooting in the Packet Tracer simulator. The students were then required to determine the problem and address obstacles to allow the network to connect and work properly in the simulation environment. Control group students were required to complete the learning activities individually, whereas experimental group students utilized cooperative learning to complete the learning activities in accordance with CPS pedagogy. The nine steps of the CPS pedagogy and their implementation in this study are presented in Table II. The resulting pre- and post-test scores of individuals on the BNCI were obtained in the same manner for students in both experimental groups.

TABLE II: MAPPING OF NINE STEPS OF CPS AND IMPLEMENTATIONS

\begin{tabular}{cl}
\hline \hline Step & A description of the implementation in this experiment \\
\hline 1. & CPS: Instructor and learners build their readiness to engage in \\
collaborative group work
\end{tabular}
In this study: Before the activity started, the teacher explained the problem and the desired target to the students and provided guidance to students regarding defining the problem and problem-solving directions, thereby establishing the learners' capabilities.

2. CPS: Either the instructor or the learners form small, heterogeneous work groups, and then the groups engage in norming processes.

In this study: The teacher divided the students into heterogeneous groups for the educational activities based on pre-test scores, with 3-5 students in each group and a total of 11 groups. The average pre-test score for each group ranged from 57-63 points.

CPS: The group engages in a preliminary process to define the problem they will work on.

In this study: Each group of students was required to cooperate to

3. complete network-troubleshooting tasks. Each group was provided with an outlined questionnaire to provide conceptual guidance for problem solving and encourage discussion among the students.

CPS: Each group defines what roles are necessary to accomplish the design plan and then assigns them.

4. In this study: Each group elected a team leader to lead the learning associated with the discussion of a single issue, devote attention to the operation-related conditions of each student in the group, and provide timely guidance to assist others.

CPS: The group engages in the primary, iterative CPS process.

In this study: Group members engaged in manipulations to test network-troubleshooting solutions and participated in group

5. discussions. Members addressed the issues of each stage to advance to the subsequent evaluation process. As the problems or activities neared completion, the students' conceptual understanding became increasingly complete.

CPS: Groups begin to finalize their solutions or projects.

In this study: After interactions through CPS teaching

6. approaches, each group began to converge on the optimal solution for the problem. Final tests of network connectivity were then performed with Packet Tracer.

CPS: The instructor and learners engage in activities to help them reflect and synthesize their experiences

In this study: Led by the team leader, the members of each group shared their experiences during the educational process, with reflection after solving the problem and confirmation that group members had completed their tasks.

CPS: The instructor and the learners assess their products and processes when appropriate.

8. In this study: Teams were recognized after they had completed their tasks. In addition, each student was required to complete the post-test questions after finishing the network troubleshooting (the post-test was identical to the pre-test).

CPS: The instructor and learners develop an activity to bring closure to the learning event.

9. In this study: The teacher provided a summary of the entire activity, enabling students to feel a sense of accomplishment regarding their participation in the learning process. 


\section{B. Participants}

The study participants were 72 undergraduate students who were enrolled in the course "An Introduction to Networks" offered by a university in northern Taiwan. The duration of this course was 16 weeks, and the curriculum objectives were to build basic network competence and skill for troubleshooting. The 34 participants in the control group included 19 men $(56 \%)$ and 15 women $(44 \%)$, whereas the 38 participants in the experimental group included 21 men (55\%) and 17 women (45\%).

\section{Research Tools}

The following research tools were utilized in this study:

- Packet Tracer simulation exercise: This simulation exercise was designed by teachers with over 10 years of network-related teaching experience to provide instruction in network concepts. The major theme of the exercise was network troubleshooting. To complete the educational activities, students were required to identify and solve a problem in the simulator environment.

- Outline questionnaire to guide the simulation exercise: By providing an outline with conceptual guidance for troubleshooting in the Packet Tracer simulation exercise, this questionnaire assisted students in the completion of the educational activities of the learning process. However, this questionnaire was only an aid to the educational activities and was not scored.

- Basic Network Concept Inventory: This inventory, which was designed by the course instructor, contains a total of 10 multiple-choice and essay questions covering basic network concepts, including 5 questions on fundamental concepts and 5 questions on troubleshooting concepts.

\section{RESEARCH RESULTS}

In this study, pre-test and post-test measurements using the BNCI were performed for the experimental and control group students. Paired-samples t-tests were used to investigate the relationship between teaching with the Packet Tracer simulator and learning outcomes. The resulting analysis of the pre- and post-test scores of the control and experimental group students is presented in Table III. The results indicated that knowledge of basic network improved in both groups of students after conducting the educational activities described above.

TABLE III: AN ANALYSIS OF PRE- AND POST-TEST SCORES FOR CONTROL AND EXPERIMENTAL GROUP STUDENTS WHO PARTICIPATED IN THE EDUCATIONAL ACTIVITIES OF THIS STUDY

\begin{tabular}{|c|c|c|c|c|c|c|}
\hline Group & Test & $\begin{array}{c}\text { Number of } \\
\text { students }\end{array}$ & Mean score & Standard deviation & $t$ & $p$ \\
\hline \multirow{2}{*}{ Control group } & Pre-test & 34 & 57.29 & 23.984 & \multirow{2}{*}{-2.719} & \multirow{2}{*}{$p=.010(<0.05)$} \\
\hline & Post-test & 34 & 62.91 & 22.348 & & \\
\hline \multirow{2}{*}{ Experimental group } & Pre-test & 38 & 57.63 & 20.898 & \multirow{2}{*}{-4.448} & \multirow{2}{*}{$p=.000(<0.001)$} \\
\hline & Post-test & 38 & 69.21 & 18.914 & & \\
\hline
\end{tabular}

Furthermore, the researchers used analysis of covariance to examine the pre- and post-test differences between the experimental and control group of some subjects. First, the homogeneity of the regression coefficients within the groups was examined. These tests resulted in an F-value of 2.054 and a p-value of 0.156 , which did not reach significance $(>0.05)$. Therefore, the relationship between the covariate (pre-test scores) and the dependent variable (post-test scores) did not change due to differing treatment standards associated with the independent variable. Thus, the homogeneity assumption for the regression coefficients of variables within groups was satisfied, and the subsequent analysis of covariance was applicable. The results of independent-samples analysis of covariance after excluding the impact of pre-test scores (the covariate) on post-test scores (the dependent variable) indicated that the impact of the independent variable on the dependent variable had an F-value of 4.327 and a p-value of 0.041 ; this impact reached the threshold for significance $(<0.05)$, indicating that participants' post-test scores varied due to the different teaching methods used in the different experimental groups. Assuming that the homogeneity assumption for regression coefficients within groups holds, the covariance analysis process indicated that the adjusted mean post-test scores were 69.02 for the experimental group and 62.75 for the control group after excluding the impact of covariance on the dependent variable and adjusting the actual post-test scores of each group based on the corresponding pre-test scores. Thus, it was evident that the post-test scores of the experimental group students were significantly better than the post-test scores of the control group students.

\section{DISCUSSION AND CONCLUSION}

Due to the abstract nature of basic network concepts, providing hands-on operations to learners to enable the construction of procedural knowledge and train actual implementation ability is an important aspect of learning relevant skills. A characteristic of computer simulation learning is that it allows for the concrete examination of phenomena that are difficult to observe in real environments. Moreover, guidance and assistance from the simulation system can help students understand these phenomena. Therefore, this study used a quasi-experimental approach to investigate whether educational activities using network simulation software were beneficial to the learning outcomes of college students enrolled in a course of basic network concepts. Furthermore, researchers divided the students into an experimental group and a control group to explore whether integrating network simulation-based learning tool with CPS teaching strategies could better enhance students' learning outcomes relative to the use of network simulation-based learning tool alone. 
The results indicated that there were significant differences in the pre- and post-test scores of the control group students who completed the educational activities using the network simulation-based learning tool, indicating that the educational process involving this network simulation-based learning tool improved learning outcomes for these students. This finding is consistent with the results of prior studies [3], [4].The experimental group students who completed the educational activities in which the network simulation-based learning tool was integrated with CPS teaching strategies also exhibited significantly different pre- and post-test scores, indicating that the design of these educational activities also improved students' learning outcomes.

In addition, researchers used analysis of covariance to test for pre- and post-test differences between the control and experimental groups. The results of this analysis demonstrated that the experimental group's post-test scores were significantly better than the control group's post-test scores. These findings illustrate that the educational activities that integrated CPS teaching strategies with network simulation were more beneficial to students than educational activities that only utilized network simulation as an instructional tool.

Therefore, the results of this study will provide teachers in relevant fields with a reference for teaching. The simple application of network simulation as an instructional tool can enhance students' learning outcomes. However, the simulated learning environment is manipulated by individual learners; thus, the use of this environment may cause students to feel a sense of isolation, and the learning process can be interrupted because students may not be able to obtain help when they encounter problems. To address this issue, the designed educational activities that integrate simulation learning and cooperative learning can achieve the objectives of teaching abstract concepts and improving students' operational capabilities. Moreover, this design allows students to share and discuss relevant topics with other students during educational activities; this aspect of the integrated approach promotes the internalization of knowledge and the development of better teamwork skills, enhancing students' adaptability and competitiveness in society

\section{REFERENCES}

[1] P. Doulai, "The role of computer simulation in electric energy systems education," in Proc. the Tomorrow's Education in Electrical Technologies; Revisited Methods and Tools for Renewed Motivation, European Power Electronics and Drive Association, pp. 159-165, 2001.

[2] L. Carabaneanu, R. Trandfir, and M. I. Mazilu, "Trends in e-learning (with Carabaneanu L., Trandafir R.)," Tampere Polytechnic, 2006, pp. 106-111.

[3] C. Goldstein, S. Leisten, K. Stark, and A. Tickle, "Using a network simulation tool to engage students in active learning enhances their understanding of complex data communications concepts," in Proc. Seventh Australasian Computing Education Conference (ACE2005), Newcastle: Australia, pp. 223-228, 2005.

[4] C. Yehezkel, M. Eliahu, and M. Ronen, "Easy CPU: Simulation-based learning of computer architecture at the introductory level," International Journal of Engineering Education, pp. 228-238, vol. 25, no. 2, 2009.

[5] B. White, "Thinker tools: Causal models, conceptual change, and science education," Cognition and Instruction, pp. 1-100, vol. 10, 1993.
[6] B. Y. White and J. R. Frederiksen, "Inquiry, modeling, and metacognition: Making science accessible to all students," Cognition and Instruction, pp. 3-118, vol. 16, 1998.

[7] L. S. Vygotsky, Mind in Society, Cambridge, MA: Harvard University Press, 1978.

[8] E. Jacob, Cooperative Learning in Context, Albany: State University of New York Press, 1999.

[9] W. M. Roth and A. Roychoudhury, "The nature of scientific knowledge, knowing and learning: The perspectives of four physics students," International Journal of Science Education, pp. 27-44, vol. 15, 1993.

[10] J. Bransford, A. Brown, and R. Cocking, How People Learn: Brain, Mind, Experience, and School, Washington D.C.: National Academy Press, 2000, pp. 3-23.

[11] C. E. Wieman, W. K. Adams, and K. K. Perkins, "PhET simulations that enhance learning," Science, pp. 682-683, vol. 322, 2008.

[12] Z. C. Zacharia and G. Olympiou, "Physical versus virtual manipulative experimentation in physics learning," Learning and Instruction, pp. 317-331, vol. 21, no. 3, 2011.

[13] Z. C. Zacharia, G. Olympiou, and M. Papaevripidou, "Effects of experimenting with physical and virtual manipulatives on students' conceptual understanding in heat and temperature," Journal of Research in Science Teaching, pp. 1021-1035, vol. 45, no. 9, 2008.

[14] D. Jonassen, Computer in Classroom. Mindtools for Critical Thinking, Englewood Clifs, NJ: Prentice-Hall, Inc., 1996.

[15] J. R. Dennis and R. J. Kansky, "Electronic slices of reality: The instructional role of computerized simulations," Instructional Computing: An Acting Guide for Educators, Glenview, Illinois: Scott, Foresman, 1984.

[16] H. Pol, E. Harskamp, and C. Suhre, "Solving physics problems with the help of computer-assisted instruction," International Journal of Science Education, pp. 451-469, vol. 27, no. 4, 2005.

[17] H. J. Pol, E. G. Harskamp, C. J. M., Suhre, and M. J. Goedhart, "The effect of hints and Model answers in a student-controlled problem-solving program for secondary physics education," Journal of Science Education and Technology, pp. 410-425, vol. 17, no. 4, 2008.

[18] K. Y. Yang, and J. S. Heh, "The impact of Internet virtual physics laboratory instruction on the achievement in physics, science process skills and computer attitudes of 10th-grade students," Journal of Science Education and Technology, pp. 451-461, vol. 16, no. 5, 2007.

[19] L. Stern, N. Barnea, and S. Shauli, "The effect of a computerized simulation on middle school students' understanding of the Kinetic molecular theory," Journal of Science Education and Technology, pp. 305-315, vol. 17, no. 4, 2008.

[20] H. C. Liu and I. H. Su, "Learning residential electrical wiring through computer simulation: The impact of computer-based learning environments on student achievement and cognitive load," British Journal of Educational Technology, pp. 598-607, vol. 42, no. 4, 2011,

[21] D. C. Frezzo, J. T. Behrens, R. J. Mislevy, P. West, and K. E. DiCerbo, "Psychometric and evidentiary approaches to simulation assessment in packet tracer software," presented at the 2009 Fifth International Conference on Networking and Services, Valencia, Spain, April 20-25, 2009.

[22] D. C. Frezzo, J. T. Behrens, and R. J. Mislevy, "Design patterns for learning and assessment: Facilitating the introduction of a complex simulation-based learning environment into a community of instructors," Journal of Science Education and Technology, pp.105-114, vol. 19, no. 2, 2010.

[23] R. M. Gagné, "Learnable aspects of problem solving," Educational Psychologist, pp. 84-92, vol. 15, no. 2, 1980.

[24] R. M. Gagné and L. J. Briggs, Principles of Instructional Design, 2nd ed., New York: Holt, Rinehart \& Winston, 1979.

[25] S. Oh and D. H. Jonassen, "Scaffolding online argumentation during problem solving," Journal of Computer Assisted Learning, pp. 95-110, vol. 23, no. 2, 2007

[26] H. I. Stromso, P. Grottum, and K. H. Lycke, "Content and processes in problem-based learning: A comparison of computer-mediated and face-to-face communication," Journal of Computer Assisted Learning, pp. 271-282, vol. 23, no. 3, 2007.

[27] L. M. Nelson, "Collaborative problem solving," Instructional-Design Theories and Models: A New Paradigm of Instructional Theory, Mahwah, N.J: Erlbaum Associates, 1999.

[28] H. Mclellan, "Situated learning in focus: Instruction to special issue," Educational Technology, pp. 5-9, vol. 33, no. 3, 1993.

[29] C. M. Achilles and S. P. Hoover, "Transforming administrative praxis: The potential of problem-based learning (PBL) as a schoo improvement vehicles for middle and high schools," presented at the 
Annual Meeting of the American Educational Research Association, New York, NY, April 8-12, 1996.

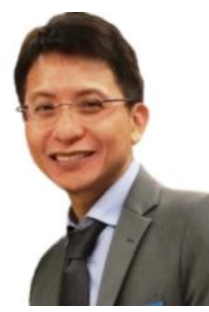

Hsin-Ke LU is the director of information management (master programs for working learners) and the CEO of School of Continuing Education at Chinese Culture University in Taiwan. He is the chairman of the Association of Continuing Education of Colleges and Universities in Taiwan and the chairman of Cisco Networking Academy. His academic interests focus on e-learning, corporate structure, lifelong learning, and information system planning (enterprise architecture).

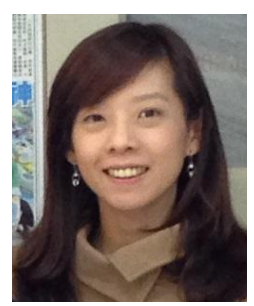

Peng-Chun LIN is an assistant professor of the Department of Information Management in Chinese Culture University. She is also the chief officer of the International Information Certification Cooperation Center at Chinese Culture University in Taiwan. Her academic interests focus on e-learning, network community development, application of simulation learning and recently on optimal network management and corporate structure. 